Trabajos de Geología, Universidad de Oviedo, 34 : 125-132 (2014)

\title{
Mapa geomorfológico del sector sur del Macizo de Las Ubiñas (Cordillera Cantábrica, NO de España)
}

\author{
Victoria Alonso \\ Departamento de Geología, Universidad de Oviedo, cl Jesús Arias de Velasco s/n, 33005 Oviedo, España \\ (e-mail:valonso@geol.uniovi.es)
}

\begin{abstract}
Resumen: Se presenta un mapa geomorfológico de la parte sur del Macizo de las Ubiñas, realizado a escala 1:1000. Este macizo, predominantemente calcáreo, con una dirección aproximada N-S, contiene varios picos de más de $2400 \mathrm{~m}$ de altura y ha sido intensamente glaciado. Además de las formas de origen glaciar, destacan en la cartografía los taludes y conos de derrubios, así como numerosos deslizamientos complejos. Una continuación de esta cartografía hacia la parte norte del macizo permitirá la interpretación de la evolución geomorfológica de esta zona.
\end{abstract}

Palabras clave: mapa geomorfológico, modelado glaciar, modelado kárstico, Macizo de la Ubiñas, Cordillera Cantábrica.

\begin{abstract}
A new geomorphological map of the southern sector of the Ubiñas massif, at a scale of $1: 1000$, is presented. This massif, a calcareous mountain alignment with an approximate N-S direction and several peaks with altitudes above $2400 \mathrm{~m}$, has been widely glaciated. The main features in the map are glacial forms as well as talus slopes, debris cones and numerous mixed slides. The continuation of this cartography to the north part of the massif will allow the interpretation of the morphological evolution of the zone.
\end{abstract}

Key words: geomorphological map, glacial landscape, karstic landforms, Ubińas Massif, Cantabrian Mountains.

Con motivo de la confección del inventario de "Lugares de Interés Geológico” promovido por el Instituto Geológico y Minero de España (2014-15), se ha elaborado un mapa geomorfológico del sector sur del Macizo de las Ubiñas a escala 1:1000 (Fig. 1) en el que se recogen los principales rasgos superficiales de la zona.

Este macizo, con una dirección aproximada NO-SE, constituye una zona elevada en la que destacan los fuertes relieves calcáreos sobre las áreas con sustrato silíceo. La mayor parte del macizo se encuentra por encima de la cota de $1700 \mathrm{~m}$ y presenta las cumbres más altas en este sector de la cordillera: El Fontán Sur, con 2414 m, y Peña Ubiña La Grande, con $2411 \mathrm{~m}$. La zona cartografiada (parte sur del macizo) pertenece a tres concejos: Quirós y Lena, en Asturias, y al municipio de San Emiliano, en León. El cordal de las Ubiñas (delineado de norte a sur por el Pico Colines, El Prau, El Siete, Peña Ubiña La Grande y Peña Ubiña Pequeña, esta última separada del resto) constituye la divisoria de aguas principal en este área; el macizo está drenado por las cabeceras de dos afluentes del río Nalón al norte, que vierte al Mar Cantábrico, y del río Luna, afluente del Duero que vierte al Océano Atlántico, al sur. En las zonas 
de cabecera, la red fluvial muestra un desarrollo irregular, con cursos discontinuos, debido principalmente a la naturaleza calcárea del sustrato, aunque también se encuentra parcialmente controlada por grandes deslizamientos.

Desde un punto de vista geológico, el Macizo de las Ubiñas se encuentra dentro del Manto del Aramo (Unidad de Ponga, Alonso et al., 2009) formando parte del zócalo varisco de la Zona Cantábrica. En el Terciario, la orogenia alpina originó la Cordillera Cantábrica, reactivando las estructuras variscas previas y creando, a su vez, alguna estructura nueva, aunque el principal efecto que tuvo esta orogenia fue el levantamiento del basamento (Alonso et al., 1996; Pulgar et al., 1999; Gallastegui, 2000). La zona de las Ubiñas destaca sobre el entorno próximo por presentar un relieve prominente de picos calcáreos de edad carbonífera (Fm. Caliza de Montaña), con altitudes cercanas o superiores a los 2400 $\mathrm{m}$, mientras que en las áreas circundantes la altitud máxima de los picos se sitúa en torno a los 2000 $\mathrm{m}$. Esta diferencia de altura, que se podría suponer resultante de la erosión diferencial de las calizas, ha sido atribuida por Martínez Abad (2007) a causas estructurales, ya que las mismas litologías en zonas próximas no forman relieves tan elevados.

La sucesión estratigráfica, aunque incompleta, abarca del Paleozoico al Cuaternario. En el Paleozoico existen dos lagunas estratigráficas: una que incluye al Ordovícico Medio y Superior y a la base del Silúrico y otra que comprende parte del Devónico Medio y Superior. El Mesozoico está representado por una serie terrígena que aflora al norte de Peña Ubiña Pequeña (Martínez Abad, 2007). En esta zona, los materiales que ocupan una mayor superficie corresponden a la Fm. Caliza de Montaña y la Fm. San Emiliano, ambas de edad carbonífera.

La zona cartografiada se encuentra dentro de un espacio protegido por la ley desde 2006: forma parte del "Parque Natural de las Ubiñas y de la Mesa". Entre los valores de este Parque, destacan los fuertes relieves, la variedad litológica, con sustratos calcáreos y silíceos, y el desarrollo de diferentes tipos de modelado: glaciar, kárstico y fluvial. Desde un punto de vista biológico, una parte importante de la superficie está ocupada por bosques maduros, principalmente hayedos y el área alberga una gran diversidad biológica, en la que se incluyen varias especies representativas de la fauna cantábrica, entre las que destaca el oso pardo. La conservación de estos valores naturales unidos al patrimonio cultural de la zona, hicieron que, en 2012, este mismo territorio fuera declarado "Reserva de la Biosfera" de la Unesco. Además este área resulta de gran interés desde un punto de vista recreativo; durante todo el ańo, el Macizo de las Ubiñas es el escenario de diversas actividades de montaña. Los pastos son aprovechados durante los meses más cálidos por los ganaderos como puertos de verano. Así mismo, presenta interés histórico, ya que en estas montańas se conservan abundantes restos de la guerra civil: nidos de ametralladora, como los situados al este de Peña Cerreos que controlaban el paso por los Puertos de Retuerto, y búnkeres, como el excavado al norte del refugio del Meicín.

Los antecedentes cartográficos sobre la morfología de la zona no son abundantes. Existe un esquema sobre la morfología glaciar (Castañón, 1983), que abarca una superficie más extensa hacia el norte que la recogida en el mapa que aquí se presenta, ya que se extiende desde el Pico Cerreos hasta los Puertos de Agüeria, por lo que no incluye el entorno de Peña Ubiña Pequeña. En un trabajo más reciente, Gallinar et al. (2014), centrándose únicamente en el sector asturiano del macizo, describen tres grandes fases en la evolución glaciar, si bien no presentan una cartografía de la zona.

\section{Mapa geomorfológico}

El mapa se realizó integrando los datos obtenidos por fotointerpretación en una aplicación SIG, sobre la base topográfica del Mapa Topográfico Nacional (MTN25 vectorial) y las ortofotografías del PNOA (Plan Nacional de Ortofotografía Aérea, 2006; tamaño de pixel $25 \mathrm{~cm}$ ), ambas disponibles en la página web del Instituto Geográfico Nacional (IGN) (http://www.ign.es/ign/main/index.do). Las fotografías aéreas consultadas corresponden a los vuelos del año 1956 (denominado "vuelo americano" del Servicio Geográfico del Ejército; Escala 1:33.000), de 1985 (vuelo nacional del IGN; Escala 1:15.000), los dos en blanco y negro, y al vuelo del año 2003 (Principado de Asturias; Escala 1:15.000) en color. 


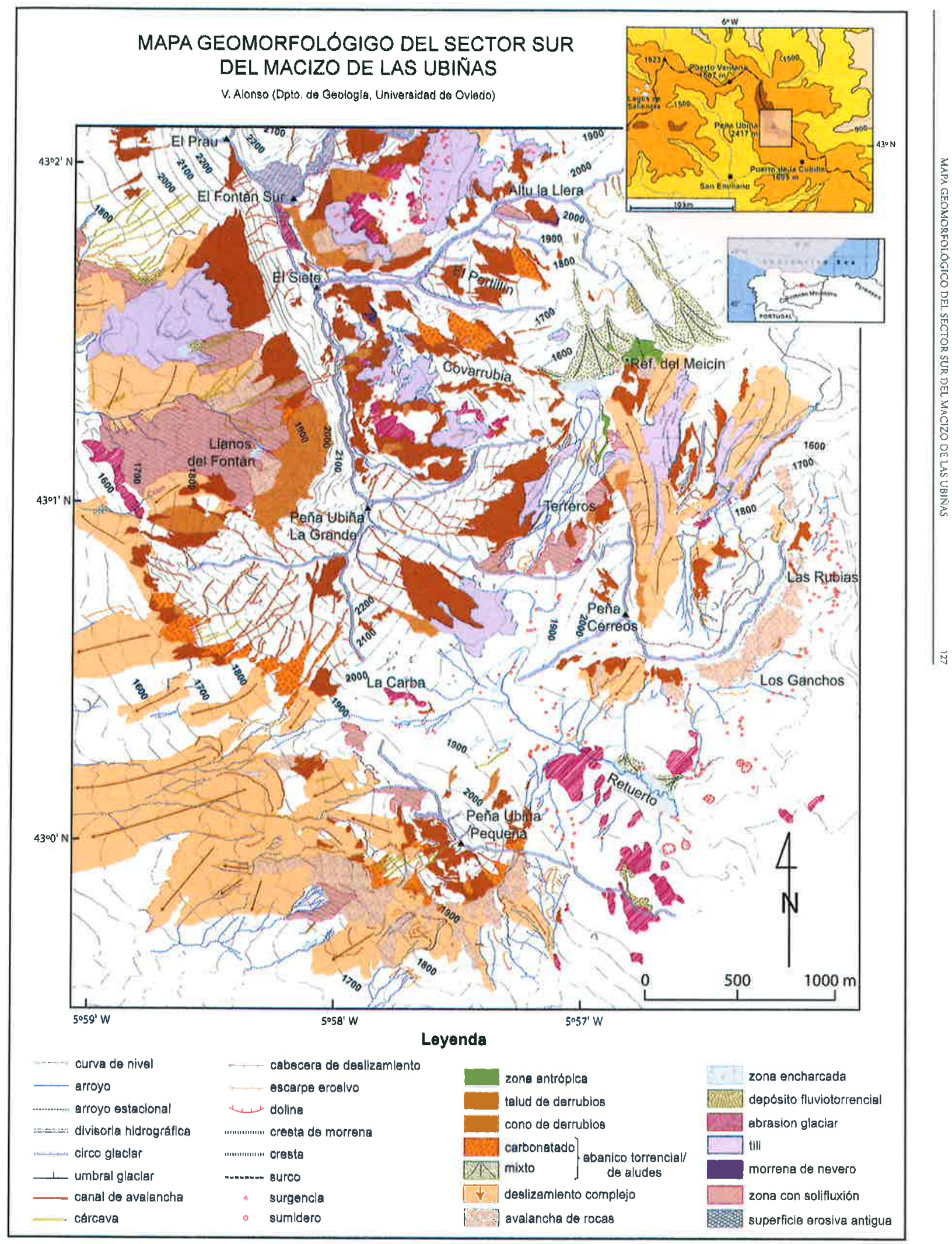

Figura 1. Mapa geomorfológico del sector sur del Maciro de las Ublíras a escala 1:1000. 
Los datos de gabinete así obtenidos fueron completados sobre el terreno en campañas de campo realizadas entre 2009 y 2014. En el campo se identificaton los rasgos de escala métrica, como las diferentes formas de solifluxión, así como la litología, forma y características superficiales de los clastos y el contenido en matriz de los depósitos, lo que contribuyó a su diferenciación.

La simbología utilizada en el mapa se adaptó, en lo posible, a la de la "Guía para la elaboración del Mapa Geomorfológico de Espańa” (Martín-Serrano et al., 2004), aunque se modificaron algunos símbolos e introdujeron otros nuevos. El mapa resultante de la aplicación SIG fue tratado con un programa de diseńo gráfico, con el fin de resaltar algunos detalles.

Para la supervisión del sustrato geológico se consultaron la hoja $\mathrm{n}^{\circ} 77$ (La Plaza) del Mapa Geológico de Espańa a escala 1:50.000 (Truyols, et al., 1982) y el trabajo de Martínez Abad (2007) sobre la geología del área entre Peńa Ubińa y el Puerto de la Cubilla.

En conjunto, el modelado de la zona responde a dos grandes tipos: glaciar y kárstico. Después de la deglaciación, la dinámica de las laderas y la abundante precipitación nival modificaron la morfología glaciar, bien retocando las formas erosivas, bien retrabajando los depósitos. Se originaron grandes deslizamientos, y otros depósitos gravitacionales, y diversas formas periglaciares, algunas aún activas en la actualidad.

\section{Elementos morfológicos}

Se han identificado formas glaciares, periglaciares, fluviororrenciales, depósitos gravitacionales de ladera (acumulaciones de derrubios de distintos tipos y deslizamientos complejos) y formas kársticas. También se han cartografiado algunas morfologías resultado de la actividad humana. De los elementos estructurales se han representado únicamente las superficies erosivas antiguas previas a la orogenia alpina, localizadas en las partes altas de las laderas, que no han sido totalmente destruidas por la actividad glaciar cuaternaria.

Entre las formas erosivas glaciares se han señalado circos poco desarrollados en la cara este de Peńa Ubi- ña La Grande y en la cara norte de Peña Ubiña Pequeña; son circos con una pendiente casi continua, sin cambios entre la pared posterior y la zona de acumulación ni tampoco hacia el frente. Hacia el norte de Peńa Ubiña La Grande, los circos cartografiados no presentan las morfologías características de este tipo de formas erosivas (pared posterior pendiente, zona de acumulación más plana, umbral frontal); la elevada actividad erosiva después de la deglaciación ha debido de modificar las formas creadas durante la glaciación. Respecto a la cara oeste del cordal que une los Fontanes con Peńa Ubiña La Grande, la acumulación de nieve debió de producirse, mediante avalanchas, al pie del talud, originándose de este modo los glaciares que fluían hacia el oeste. Los depósitos de till no se reconocen en la parte suroeste del mapa, aunque es posible que algunos hayan sido retrabajados por la erosión y estos materiales se encuentren ahora formando parte de avalanchas de rocas y/o de deslizamientos complejos. Se han marcado crestas de morrenas (laterales y frontales) en alguno de los depósitos de till, que muestran un retroceso escalonado del hielo (Fig. $2 \mathrm{~A}, \mathrm{C}, \mathrm{G}$ y H).

Respecto a las formas periglaciares, en materiales lutíticos predominan las formas de solifluxión. En materiales homogéneos, sobre sustrato lutítico o en tills, se desartrollan terracitas; se han identificado en los tills de las Cinchas del Planón (al sureste de El Siete) y en las lutitas de los Llanos del Fontán. Cuando existen bloques de caliza entre materiales más finos se pueden dar fenómenos de solifluxión retenida (la parte posterior del bloque queda embebido en el suelo, mientras que el frente está al descubierto) y también formarse bloques aradores cuando el levantamiento del suelo es más profundo; buenos ejemplos de ambos se encuentran en La Guariza, al sur del refugio del Meicín. En algunos casos se llegan a formar lóbulos de solifluxión sobre lutitas (Fig. 2 D) como los que se encuentran en Terreros, al pie de Peña Ubińa La Grande o pequeńos lóbulos sobre depósitos de ladera (como en el abanico del valle de Covarrubia, Fig. 2 A). Por lo que respecta a formas periglaciares de gran tamańo, se ha encontrado una morrena de nevero a unos $1950 \mathrm{~m}$ de altitud, al sur del Altu la Llera con una orientación sureste, y otra en la cabecera del valle de Covarrubia, al sur de EI Siete. Es posible que algunas de las morrenas más altas de Cuapalacio (en el borde norte de este mapa, 
fuera del análisis de esta parte sur del macizo) también se correspondan con morrenas de nevero. No se descarta que la alternancia de crestas y surcos concéntricos representados al pie de un talud en la zona de Los Ganchos sea un pequeño glaciar rocoso.

La red fluvial, fuertemente condicionada por el sustrato, no está bien jerarquizada. En estas zonas altas, la mayoría de los cursos fluviales son estacionales, ya que están alimentados por aguas de fusión nival o por fuertes lluvias. Sobre sustrato calcáreo son frecuentes las surgencias y los sumideros, como el de la Vega del Meicín o los del Valle de Retuerto (Fig. 2 B) cuyo nombre hace referencia a los cursos meandriformes que terminan sumiéndose en varios puntos; la zona de Las Rubias destaca por tener una elevada concentración de sumideros, algunos de gran tamaño en superficie. Donde hay grandes deslizamientos, como al oeste de Peña Ubiña Pequeña, los arroyos se adaptan a los bordes de las masas deslizadas, aunque en algunas partes también se infiltran a través de estos depósitos. En las paredes escarpadas de caliza se forman canales de avalancha de nieve que, en ocasiones, se continúan hacia abajo con torrenteras o cárcavas que erosionan los depósitos situados al pie de los taludes o que se encuentran encajadas en materiales lutíticos, como sucede en la cara oeste al pie de Peña Ubiña La Grande. Los dos casos pueden interpretarse como cabeceras de deslizamientos asociados a las inestabilidades provocadas por una erosión intensa.

En los depósitos de ladera se ha distinguido entre taludes y conos de derrubios; ambos se sitúan al pie de paredes escarpadas pero se diferencian en su forma en planta. Mientras los taludes de derrubios forman mantos que regularizan las pendientes, los conos se sitúan al pie de canales de avalancha que los alimentan desde un punto donde se forma el ápice, abriéndose el depósito hacia abajo dando forma de abanico en planta (Fig. 2 G). En el caso de que los conos sean coalescentes, pueden dar un conjunto similar a los taludes de derrubios, el mejor ejemplo se encuentra en al noroeste de Peña Ubiña La Grande (Fig. 2 E). Al pie de los escarpes en las partes altas, y también sobre las laderas, se han formado, además, abanicos torrenciales y de aludes. Se diferencian de los conos de derrubios en que presentan una mayor participación nival y de la arroyada en su formación, lo que provoca que tengan la superficie incidida por pequeños canales de alimentación pluvionival. Se han distinguido dos tipos en función de la litología del área fuente y del contenido en matriz: carbonatados y mixtos. Los abanicos carbonatados se encuentran principalmente al suroeste de Peña Ubiña La Grande, al sur de Peña Ubiña Pequeña y en los valles del Portillín y de Covarrubia (Fig. 2 A). Los abanicos de aludes de Covarrubia constituyen uno de los mejores ejemplos y, en el situado más al este, como se ha señalado anteriormente, se pueden apreciar procesos de solifluxión. Cuando los abanicos reciben aportes terrígenos, presentan un contenido en matriz más alto y, en general, un mayor desarrollo; en estos la acción torrencial es más importante que en los carbonatados.

Además de estos depósitos, al pie de las laderas se han encontrado avalanchas de rocas y deslizamientos complejos. La avalanchas de rocas son depósitos formados por grandes bloques que se encentran cubriendo un talud, un buen ejemplo se encuentra al pie del escarpe oriental de El Puerto Cerreo (desde la zona de los Ganchos hacia el norte). En otros casos, se sitúan al frente de taludes o de conos de derrubios formando depósitos muy superficiales constituidos por bloques de gran tamaño que se han desplazado alejándose del frente rocoso debido a su mayor masa.

Sobre sustrato lutítico o arcilloso, como los materiales de la Fm. San Emiliano, son frecuentes los deslizamientos complejos, que, en algunos casos, afectan también a depósitos glaciares. En ocasiones, las cabeceras de los deslizamientos se confunden con los escarpes erosivos; en muchos casos la erosión de los cursos estacionales en materiales blandos provoca inestabilidades en las pendientes que se originan con la profundización de los cauces, como sucede en los Llanos del Fontán, al pie de la vertiente noroeste de Peña Ubiña La Grande. Sobre estas mismas litologías, y en suelos homogéneos, existen abundantes cicatrices semicirculares marcadas por una diferencia en la vegetación, abiertas ladera abajo, de tamaño métrico, que se corresponden con cabeceras de pequeños deslizamientos; se han identificado, principalmente, en las proximidades de La Carba, al sur de Peña Ubiña La Grande.

La mayor concentración de avalanchas de rocas y de deslizamientos se localiza en la parte sur y suroes- 
te de Peña Ubińa Pequeña, donde toda la ladera se encuentra removida. Esta cara de Peña Ubiña ha experimentado un desmoronamiento muy importante (Fig. 2 F), encontrándose grandes bloques de Caliza de Montańa sobre la Fm. San Emiliano, incluso a distancias superiores a $1 \mathrm{~km}$ de la cumbre.

Tanto en los deslizamientos, como en las avalanchas de rocas, se han marcado algunas crestas, y surcos, representadas con un color pardo, para diferenciarlas claramente de las crestas morrénicas, en tono malva. Alguna de estas crestas y surcos serían compatibles con la interpretación del depósito en el que se encuentran como un glaciar rocoso (ej. avalancha en el escarpe oriental de El Puerto Cerreo, zona de Los Ganchos) mientras que otras podrían corresponder a formas de flujo o bien reflejar irregularidades en el sustrato.

En cuanto al modelado kárstico, como se ha visto con gran influencia sobre la red fluvial, destaca la abundancia de surgencias y de sumideros en todas las zonas con afloramientos calcáreos, incluidas las partes altas, aunque aquí predominan las formas glaciares y periglaciares. Se han encontrado pequeñas dolinas sobre la Fm. Caliza de Montaña al este de los Puertos de Retuerto. Los lapiaces, formas a pequeña escala desarrolladas después de la deglaciación, se encuentran principalmente en las áreas más bajas, aunque también son frecuentes en las superficies pu- lidas por el hielo de las zonas más altas y en algunos clastos que forman parte de los depósitos.

\section{Algunas consideraciones finales}

Un análisis somero de este mapa muestra dos rasgos a destacar: la poca superficie ocupada por los tills y el desmantelamiento de Peña Ubiña Pequeña. Durante el último ciclo glaciar el macizo debió de estar, en gran parte, cubierto por el hielo. En Babia Alta, unos $20 \mathrm{~km}$ hacia el oeste, y con altitudes inferiores a las de esta zona, se ha probado la existencia de un pequeño casquete de hielo (Alonso y Suárez Rodríguez, 2004). Por extensión, y teniendo en cuenta la topografía y la distribución de los depósitos de till, se puede afirmar que en Ubiña hubo un glaciarismo de tipo alpino combinado con pequeñas plataformas de hielo similares a las originadas en Babia. Sin embargo, la falta de materiales competentes en algunas zonas y el que los aportes supraglaciares debieron de ser poco importantes pueden explicar el escaso volumen de los depósitos de till. Además, los tills pueden haber sido retrabajados, encontrándose ahora formando parte o por debajo de los depósitos de talud, de las avalanchas o de los deslizamientos complejos.

Respecto a Peña Ubiña Pequeña (Fig. 2 F) resulta muy llamativo el aspecto de su cara oeste, totalmente

Figura 2. A (página siguiente). Taludes y conos de derrubios y abanicos de derrubios entre Peña Ubiña La Grande y el Picu La Palazana, vista hacia el norte. En el centro, en primer plano, morrena glaciar en Terreros. El abanico marcado con un asterisco, en el valle de Covarrubia, muestra modificaciones en superficie por procesos de solifluxión; hacia arriba se puede apreciar como el ápice del abanico enlaza con el canal que lo alimenta, marcado por la nieve. B. Vista hacia el sureste de los Puertos de Retuerto. Cursos meandriformes y meandros abandonados. La corriente se sume en el extremo sur del valle. A la derecha, afloramientos de caliza pulidos por el hielo situados al sureste de Peña Ubiña Pequeña. C. Vista hacia el sur de las morrenas laterales situadas al SE del refugio del Meicín. El pico más alto a la derecha, Peńa Cerreos con $2111 \mathrm{~m}$, presenta grandes avalanchas de rocas en su vertiente oriental. D. Lóbulos de solifluxión en la parte alta de Terreros, al pie de Peńa Ubiña La Grande (vista hacia el suroeste). Los relieves de los bordes, y en algunos casos la diferencia de vegetación de los frentes, indican actividad actual. E. Vista hacia el sureste del Macizo de las Ubiñas, desde el Picu Colines a Peña Ubiña La Grande (PUG). La Fm. Caliza de Montaña forma escarpes rocosos que destacan sobre la Fm. San Emiliano. Al pie de estos escarpes se forman taludes de derrubios y conos de derrubios que enlazan, hacia arriba, con canales de avalancha. Hacia la derecha, separada de este cordal, Peña Ubiña Pequeña (PUP). El asterisco señala el mismo punto que en la foto $\mathrm{H}$, situado por debajo de Los Castillinos. F. Cara suroeste de Peña Ubiña Pequeña, donde se puede observar el desmantelamiento de la misma. Parte de las calizas se encuentran formando grandes avalanchas de rocas que se extienden ladera abajo cubriendo la Fm. San Emiliano. G. Vista hacia el sureste de Peña Cerreos desde Las Cinchas del Planón. Morrena glaciar, remarcada por la nieve, en primer plano. Taludes y conos de derrubios al pie de la cara noreste de Peńa Ubiña La Grande (el asterisco señala un cono de derrubios). H. Morrenas glaciares, formando sucesivos arcos de retroceso, al pie del cordal de las Ubińas en su parte occidental (vista hacia el noroeste). Los glaciares en esta zona se habrían formado por acumulación de nieve caída por avalanchas desde las paredes escarpadas que se pueden apreciar a la derecha de la fotografía. 

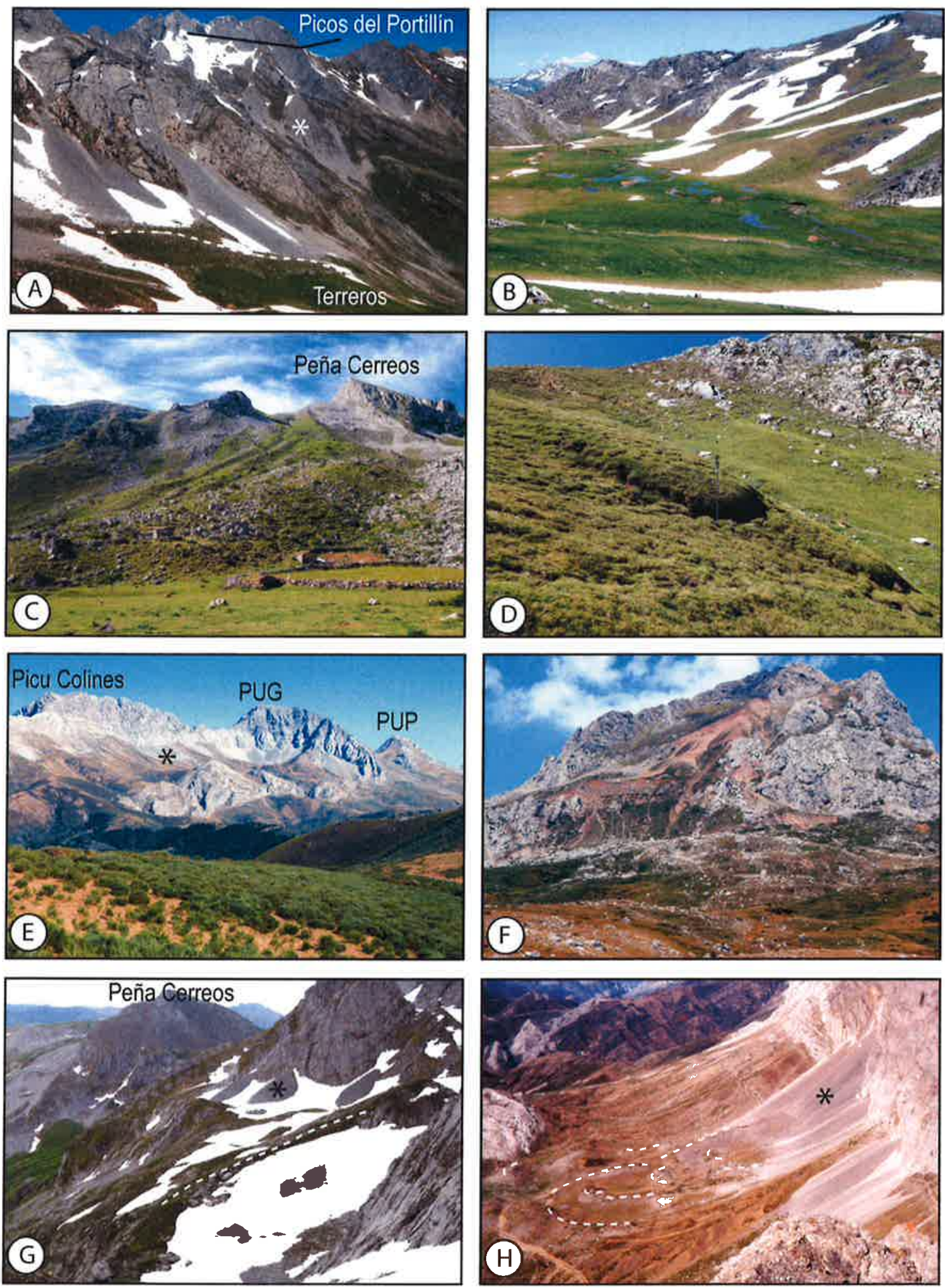
cubierta de taludes de derrubios, de deslizamientos y de avalanchas de rocas. No se descarta que esto pueda deberse a una intensa fracturación de las calizas que la forman, aunque también la relación entre la Fm. Caliza de Montaña y la Fm. San Emiliano, hacia el oeste, parece haber influido en este desmoronamiento, habiéndose observado grandes avalanchas de rocas, en otras zonas de la Cordillera Cantábrica, donde la serie se encuentra muy verticalizada o ligeramente invertida, como al este de Entrago (Asturias) o al noreste de Villamanín (León).

Cabe señalar, por otro lado, que a pesar de las altitudes que se alcanzan en este área, no se hayan encontrado glaciares rocosos (exceptuando la forma dudosa situada al norte de Los Ganchos), las mesoformas periglaciares más características de la cordillera. En Yegüero (unos $14 \mathrm{~km}$ al suroeste de Peña Ubiña La Grande) existe un glaciar rocoso de gran tamaño, y otros más pequeños, formados por bloques calcáreos. Sin embargo, la cuarcita es la litología dominante en los glaciares rocosos de la Cordillera Cantábrica, por lo que parece que en esta cordillera existe un cierto control litológico para su formación.

Una continuación de esta cartografía hacia la parte norte del macizo permitirá, en un futuro próximo, realizar un análisis de conjunto de la evolución geomorfológica de esta zona.

\section{Agradecimientos}

A Ángela Suárez y a Óscar Merino por sus aportaciones y comentarios durante la realización de este trabajo. Agradezco a Luis Alfonso Fernández, a Mariate Alonso-Vega y a Javier Nuevo su inestimable compañía en el campo. A Rosana Menéndez Duarte, y a otro revisor anónimo, cuyas aportaciones contribuyeron a la mejora de este trabajo.

\section{Bibliografía}

Alonso J.L., Marcos A. y Suárez, A. (2009): Paleogeographic inversion resulting from large out of sequence breaching thrusts: The Leon Fault (Cantabrian Zone, NW Iberia). A new picture of the external Variscan Thrust Belt in the Ibero-Armorican Arc. Geologica Acta, 7 (4): 451-473.

Alonso, J.L., Pulgar, F.J., García-Ramos, J.C. y Barba, P. (1996): Tertiary Basins and Alpine Tectonics in the Cantabrian Mountains. En: Friend, P.F. y Dabrio, C.J. (eds.). Tertiary Basins in Spain: The stratigraphic record of cristal kinematics. Cambridge University Press: 214-227.

Alonso, V. y SuÁrez Rodríguez, A. (2004): Evidencias geomorfológicas de la existencia de un pequeño casquete glaciar en la Comarca de Babia Alta (Cordillera Cantábrica). Revista de la Sociedad Geológica de España, 17: 61-70.

Castañón, J.C. (1983): El glaciarismo cuaternario del Macizo de Ubiña (Asturias-León) y su importancia morfológica. Ería, 1983: 3-49.

Gallastegui, J. (2000): Estructura de la cordillera y margen continental cantábricos: perfiles ESCI-N. Trabajos de Geologia, 22: 1-221.

Gallinar, D., Ruiz-Fernández, J., Poblete, M.A., Fernández, A. García, C.; Beato, S. y Marino, J.L. (2014): Morfología y evolución glaciar en el sector asturiano del Macizo de las Ubiñas. Actas de la XIII Reunión Nacional de Geomorfología, Cáceres: 543-546.

Martín-Serrano, A., Salazar, A., Nozal, F. y Suárez, A. (2004): Mapa Geomorfológico de España a escala 1:50.000. Guía para su elaboración. Instituto Geológico y Minero de España, Madrid: 128 p.

Martínez Abad, I. (2007): Geología del área situada entre Peña Ubiña y el Puerto de La Cubilla (Zona Central de la Cordillera Cantábrica). Trabajo fin de Master, Universidad de Oviedo: 58 p.

Pulgar F.J., Alonso, J.L., Espina, R.G. y Marín, J.A. (1999): La deformación alpina en el basamento varisco de la Zona Cantábrica. Trabajos de Geología, 21: 283-294.

Truyols, J., Arbizu, M., García-Alcalde, J.L., GarcíaLópez, S., Martínez Chacón, M.L., Méndez-Bedia, I., Méndez-Fernández, J.R., Sánchez de Posada, L.C., Soto, F., Truyols-Massoni, M., Villa, E., Marcos, A., Pérez-Estaún, A., Pulgar, J.A., Bastida, F., Aller, J. y Lorenzo, P. (1982): Memoria del Mapa Geológico de España 1:50.000, Hoja 77 (La Plaza, Teverga). Instituto Geológico y Minero de España, Madrid: 64 p. 